\title{
Butorphanol, a synthetic opioid, sensitizes ABCB1-mediated multidrug resistance via inhibition of the efflux function of ABCB1 in leukemia cells
}

\author{
JING WEN $^{1 *}$, TAO ZHANG $^{1 *}$, ZHI-MING SHAN ${ }^{1}$, MIN-YUE QI $^{2}$, HUAN-HUAN XIU ${ }^{1}$, \\ LEI LIU ${ }^{3}$, SHI-ZHE WU ${ }^{2}$, ZHEN JIA ${ }^{3}$ and KANG-QING XU ${ }^{1}$ \\ ${ }^{1}$ Department of Anesthesiology, First Affiliated Hospital and ${ }^{2}$ School of Life Sciences, Sun Yat-Sen University, Guangzhou, \\ Guangdong 510080; ${ }^{3}$ Department of Anesthesiology, Qinghai University Affiliated Hospital, \\ Xining, Qinghai 810001, P.R. China
}

Received March 11, 2015; Accepted May 5, 2015

DOI: 10.3892/or.2015.4052

\begin{abstract}
Multidrug resistance (MDR) remains a formidable challenge in the use of chemotherapy and represents a powerful obstacle to the treatment of leukemia. ATP-binding cassette subfamily B member 1 (ABCB1) is a recognized factor which causes MDR and is closely related to poor outcome and relapse in leukemia. Ongoing research concerning the strategy for inhibiting the abnormally high activity of the ABCB1 transporter is critically needed. In the present study, we sought to elucidate the interaction between ABCB1 transporter and butorphanol. Our results showed that butorphanol significantly antagonized ABCB1-mediated drug efflux and increased the intracellular drug concentration by inhibiting the transport activity of ABCB1 in leukemia cells. Mechanistic investigations demonstrated that butorphanol did not alter the protein expression or localization of ABCB1 in HL60/VCR and K562/ADR cells. Furthermore, homology modeling indicated that butorphanol could fit into the large drug-binding cavity of $\mathrm{ABCB} 1$ and form a binding conformation. In conclusion, butorphanol reversed the ABCB1-mediated MDR in leukemia cells by directly suppressing the efflux activity of ABCB1.
\end{abstract}

\section{Introduction}

The optimal use of chemotherapeutics, which allows patients to achieve a completely hematologic remission, is an effective and

Correspondence to: Professor Zhen Jia, Department of Anesthesiology, Qinghai University Affiliated Hospital, Xining, Qinghai 810001, P.R. China

E-mail:projia_81000@163.com

Professor Kang-Qing Xu, Department of Anesthesiology, First Affiliated Hospital, Sun Yat-Sen University, Guangzhou, Guangdong 510080, P.R. China

E-mail:proxu_510080@163.com

*Contributed equally

Key words: multidrug resistance, ABCB1, opioid, butorphanol, combined chemotherapy crucial strategy for leukemia patients (1). However, only $40 \%$ of patients achieve a 5-year survival (2), since most patients relapse quickly after withdrawal of anticancer drugs. What is worse, advanced stage patients frequently develop resistance to drugs while receiving treatment. Intrinsic and acquired multidrug resistance (MDR) is a phenomenon whereby tumor cells are resistant to structurally and mechanistically distinct classes of compounds. MDR remains a major impediment to the decrease in relapse rates in leukemia patients (3). At present, one of the most recognized mechanisms of MDR is overexpression of ATP-binding cassette (ABC) transporters which reduce intracellular drug concentrations leading to cancer cell resistance. Among the ABC family proteins, ABC subfamily B member 1 (ABCB1) is closely associated with recurrence and a poor therapeutic response in leukemia patients (4).

ABCB1 (also named P-glycoprotein or MDR1), which is a $170-\mathrm{kDa}$ transporter, consists of two nucleotide binding domains (NBDs) and two transmembrane domains (TMDs) (5). Leukemia cells often express a high level of ABCB1 and more than $50 \%$ of conventional chemotherapeutics undergo ABCB1-mediated efflux (6). Accordingly, ABCB1 is regarded as an indicator of malignancy. Interfering with the activity of ABCB1 may effectively circumvent ABCB1-mediated drug resistance and improve the clinical efficacy of leukemia chemotherapy (7). Currently, numerous studies have sought to identify and develop effective and safe inhibitors of the ABCB1 transporter. Unfortunately, most studies have not confirmed sufficient efficacy or have been terminated due to the nonspecific toxicity associated with conventional chemotherapy drugs (8). The preliminary findings have aroused intensive interest to tackle these hurdles. Exploring natural ingredients or identifying potential sensitizers from a list of approved drugs in the clinic provide a promising approach for the development of ABCB1-modulating compounds. Butorphanol belongs to a prototypically mixed agonist-antagonist opioid analgesic with a weakly competitive $\mu$-receptor agonist and strong $\kappa$-receptor agonist. Because of its compounded agonistantagonist functions, butorphanol owns less incidences of respiratory depression and pruritus but more balance anes- 
thesia. At present, butorphanol has been frequently applied in postoperative analgesia, labor analgesia and cancer analgesia, including malignant leukemia (9-11).

In the present study, we demonstrated that butorphanol, at a clinically used dose, obviously increased the chemosensitivity of drug-resistant leukemia cells to conventional anticancer drugs by directly inhibiting the efflux activity of ABCB1.

\section{Materials and methods}

Chemicals and reagents. Butorphanol was purchased from Jiangsu Hengrui Pharmaceutical Co. (Jiangsu, China). Doxorubicin (DOX), verapamil (VRP), cisplatin, vincristine (VCR) and other chemicals were purchased from Sigma Chemical Co. (St. Louis, MO, USA). Monoclonal antibodies against ABCB1 (sc-55510) were obtained from Santa Cruz Biotechnology, Inc. (Santa Cruz, CA, USA). 1-(4,5-Dimethylthiazol-2-yl)-3,5diphenylformazan (MTT) was purchased from Zhongshan Golden Bridge Biotechmology (Beijing, China).

Cell lines and cell culture. HL60 and HL60/VCR cell lines were purchased from the Institute of Hematology, the Chinese Academy of Medical Sciences (Tianjin, China). K562 and K562/ADR cell lines were purchased from the International Medical Center of the First Central Hospital of Tianjin (Tianjin, China). HL60/VCR and K562/ADR cells were vincristine- and adriamycin-selected ABCB1-overexpressing cells, respectively (12). All of the cell lines were maintained in Dulbecco's modified Eagle's medium (DMEM) and RPMI-1640 medium with $10 \%$ fetal bovine serum (FBS) and $1 \%$ antibiotic solution (penicillin-streptomycin) at $37^{\circ} \mathrm{C}$ in a humidified atmosphere of $5 \% \mathrm{CO}_{2}$ (11). All cells were grown in drug-free culture medium for 2 weeks before the assay.

Cytotoxicity assay. The aim of the MTT assay was to evaluate the sensitivity of leukemia cells to the drugs. Cells were seeded in 96-well plates and allowed to attach overnight. Afterwards, the cells were preincubated with or without butorphanol and verapamil for $1 \mathrm{~h}$, and then various concentrations of chemotherapeutic drugs were added into the designated wells. After $68 \mathrm{~h}$, MTT was added into the wells for an additional 4-h incubation after which the yellow-colored MTT changed into dark-blue formazan crystals (13). Subsequently, the medium was discarded, and $120 \mu \mathrm{l}$ of dimethylsulfoxide (DMSO) was added to each well. The absorbance was determined at $655 \mathrm{~nm}$ using a Model 550 microplate reader (Bio-Rad, Hercules, CA, USA). The concentration required to inhibit cell growth by $50 \%\left(\mathrm{IC}_{50}\right)$ was calculated from survival curves using the Bliss methods (14). The resistance-fold was calculated by dividing the $\mathrm{IC}_{50}$ value for the MDR cells with or without inhibitor by that of the parental cells without inhibitor.

DOX accumulation. The intracellular DOX accumulation in the ABCB1-overexpressing cells (HL60/VCR) and their parental sensitive cells (HL60) was examined by flow cytometry. The logarithmically growing cells were treated with 1,2 and $4 \mu \mathrm{M}$ butorphanol and $5 \mu \mathrm{M}$ verapamil at $37^{\circ} \mathrm{C}$ for $3 \mathrm{~h}$ (15). Then DOX (terminal concentration $10 \mu \mathrm{M}$ ) was added to the designated wells followed by incubation for $30 \mathrm{~min}$ and $3 \mathrm{~h}$, respectively. The cells were then collected, centrifuged and washed twice with cold phosphate-buffered saline (PBS). Cells were resuspended in $400 \mathrm{ml}$ PBS and then analyzed by flow cytometry (Cytomics FC 500; Beckman Coulter, USA). Verapamil, which is known as an ABCB1 inhibitor, was used as a positive control. The relative values were calculated by dividing the fluoresence intensity of each measurement by that of the negative-control cells $(16,17)$.

Western blotting. Cell extracts were collected in cell lysis buffer for $20 \mathrm{~min}$ on ice, and then the cells were centrifuged at $12,000 \mathrm{rpm}$ at $48^{\circ} \mathrm{C}$ for $15 \mathrm{~min}$. Equal amounts of proteins were resolved by SDS-PAGE and transferred onto nitrocellulose membranes. Following blocking in 5\% non-fat milk in Tris-buffered saline and Tween-20 (TBST) buffer for $2 \mathrm{~h}$ at room temperature, the cells were incubated with appropriately diluted primary antibodies overnight at $4^{\circ} \mathrm{C}$ (18). The membranes were then washed thrice with TBST buffer and incubated with HRP-conjugated secondary antibody at a 1:5,000 dilution for $2 \mathrm{~h}$ at room temperature. After being washed thrice with TBST buffer, the protein antibody complex was visualized using the enhanced Phototope ${ }^{\mathrm{TM}}-\mathrm{HRP}$ detection kit (Cell Signaling, USA) and exposed to a Kodak medical X-ray processor (Carestream Health, USA). The expression of GAPDH was used as a loading control. The protein expression level was quantified using gray value analysis software (17).

Immunofluorescence staining. For immunocytochemical analysis, the cells were seeded in 24-well plates, and butorphanol at $4 \mu \mathrm{M}$ was added to the wells after overnight culture. After $72 \mathrm{~h}$ of incubation, the cells were washed with PBS and fixed with $4 \%$ paraformaldehyde for $15 \mathrm{~min}$ at room temperature and then rinsed with PBS three times (19). The cells were treated with a monoclonal antibody against ABCB1 (1:500) (Sigma Chemical Co.) and incubated overnight. Alexa Fluor 488 goat anti-mouse IgG was added and cultured for $1 \mathrm{~h}$. Immunofluorescence images were captured with an inverted microscope (Olympus IX70; Olympus, Center Valley, PA, USA) with IX-FLA fluorescence and a CCD camera (20).

Docking simulation. All docking calculations were performed using the 'Extra Precision' (XP) mode of Glide program v5.5 (Schrödinger, Inc., New York, NY, USA, 2009) and the default parameters. The top scoring pose-ABCB1 complex was then subjected to energy minimization using MacroModel program v9.7 using the OPLS-AA force field and used for graphical analysis. All computations were carried out on a Dell Precision 470n dual processor with Linux OS (Red Hat Enterprise WS 4.0) (21).

Statistical analysis. All experiments were repeated at least three times, and the Student's t-test was used to determine differences. Statistical significance was determined at $\mathrm{p}<0.05$.

\section{Results}

Reversal effects of butorphanol on ABCB1-overexpressing leukemia cells. In order to investigate the reversal effects of butorphanol, we initially evaluated its cytotoxicity. The results are shown in Fig. 1A and B. The intrinsic cytotoxic effect of butorphanol on various cell lines was examined by MTT assay. 
Table I. Effect of butorphanol on reversing ABCB1-mediated MDR to doxorubicin (DOX), verapamil, cisplatin and vincristine (VCR) in the sensitive (K562) and drug-resistant (K562/ADR) cells.

\begin{tabular}{lcc}
\hline & \multicolumn{2}{c}{$\mathrm{IC}_{50} \pm \mathrm{SD}^{\mathrm{a}}(\mu \mathrm{M})($ resistance fold $)$} \\
\cline { 2 - 3 } Treatment & $\mathrm{K} 562$ & $\mathrm{~K} 562 / \mathrm{ADR}$ \\
\hline DOX & $0.154 \pm 0.015(1.00)^{\mathrm{b}}$ & $7.412 \pm 0.117(48.13)$ \\
+Butor 1 $(\mu \mathrm{M})$ & $0.149 \pm 0.008(0.97)$ & $3.32 \pm 0.094(21.58)^{\mathrm{c}}$ \\
+Butor 2 & $0.155 \pm 0.014(1.01)$ & $2.32 \pm 0.037(15.04)^{\mathrm{c}}$ \\
+Butor 4 & $0.146 \pm 0.001(0.95)$ & $1.482 \pm 0.021(9.63)^{\mathrm{c}}$ \\
+Verapamil 5 & $0.153 \pm 0.011(0.99)$ & $1.457 \pm 0.018(9.42)^{\mathrm{c}}$ \\
VCR & $0.030 \pm 0.002(1.00)^{\mathrm{b}}$ & $3.252 \pm 0.381(108.4)$ \\
+Butor 1 $(\mu \mathrm{M})$ & $0.029 \pm 0.001(0.98)$ & $1.47 \pm 0.172(49.03)^{\mathrm{c}}$ \\
+Butor 2 & $0.029 \pm 0.001(0.98)$ & $1.08 \pm 0.105(36.00)^{\mathrm{c}}$ \\
+Butor 4 & $0.031 \pm 0.002(1.03)$ & $0.62 \pm 0.081(20.68)^{\mathrm{c}}$ \\
+Verapamil 5 & $0.029 \pm 0.001(0.97)$ & $0.61 \pm 0.085(20.08)^{\mathrm{c}}$ \\
Cisplatin & $1.63 \pm 0.174(1.00)^{\mathrm{b}}$ & $2.35 \pm 0.481(1.43)$ \\
+Butor 1 $(\mu \mathrm{M})$ & $1.52 \pm 0.165(0.93)$ & $2.40 \pm 0.427(1.47)$ \\
+Butor 2 & $1.63 \pm 0.152(1.00)$ & $2.34 \pm 0.445(1.44)$ \\
+Butor 4 & $1.60 \pm 0.149(0.98)$ & $2.27 \pm 0.385(1.39)$ \\
+ Verapamil 5 & $1.55 \pm 0.161(0.95)$ & $2.28 \pm 0.392(1.30)$ \\
\hline
\end{tabular}

${ }^{\mathrm{a}} \mathrm{IC}_{50}$ values are represented as mean $\pm \mathrm{SD}$ of three independent experiments performed in triplicate. ${ }^{b}$ The fold reversal of MDR (values given in parentheses) was calculated by dividing the $\mathrm{IC}_{50}$ values of substrate in drug-resistant cells in the absence or presence of inhibitor or K562 cells with inhibitor, by the $\mathrm{IC}_{50}$ value of $\mathrm{K} 562$ cells without inhibitor. ${ }^{c} \mathrm{p}<0.01$, significantly different from values obtained in the absence of inhibitor. ABCB1, ATP-binding cassette subfamily B member 1; MDR, multidrug resistance; SD, standard deviation; Butor, butorphanol.

Notably, the results showed that butorphanol at $4 \mu \mathrm{M}$ had no obviously cytotoxic effect on all cell lines used in the assay, and $>85 \%$ cells survived. The $\mathrm{IC}_{50}$ values were $1.76 \pm 0.09$, $1.64 \pm 0.1,1.89 \pm 0.14$ and $1.43 \pm 0.09 \mu \mathrm{mol} / 1$ for the K562, K562/ ADR, HL60 and HL60/VCR cell lines, respectively. Thus, butorphanol at $4 \mu \mathrm{M}$ was chosen as the maximum working concentration for further reversal assay.

Based on the above concentration, the $\mathrm{IC}_{50}$ values of DOX, VCR and cisplatin in resistant cells (HL60/VCR and K562/ADR) and sensitive cells (HL60 and K562) without or combined with different concentrations of butorphanol, respectively, are provided in Tables I and II. Butorphanol, at $4 \mu \mathrm{M}$, obviously decreased the $\mathrm{IC}_{50}$ values of $\mathrm{ABCB} 1$ substrates DOX and VCR in the ABCB1-overexpressing HL60/VCR and K562/ADR cells, while butorphanol did not change the $\mathrm{IC}_{50}$ values of these chemotherapeutic drugs in the parental HL60 and K562 cells. Meanwhile, the reversal effects were similar to verapamil at $5 \mu \mathrm{M}$ which is a well-known ABCB1 inhibitor. Yet, butorphanol did not affect the $\mathrm{IC}_{50}$ values of cisplatin in both the sensitive and resistant cells. Cisplatin is not a substrate of ABCB1. These results showed that butorphanol at $4 \mu \mathrm{M}$ was able to reverse resistance to DOX and VCR in the HL60/VCR and K562/ADR cells. Yet, in the parental HL60
Table II. Effect of butorphanol on reversing ABCB1-mediated MDR to doxorubicin (DOX), verapamil, cisplatin and vincristine (VCR) in the sensitive (HL60) and drug-resistant (HL60/ VCR) cells.

\begin{tabular}{lcc}
\hline & \multicolumn{2}{c}{$\mathrm{IC}_{50} \pm \mathrm{SD}^{\mathrm{a}}(\mu \mathrm{M})($ resistance folds $)$} \\
\cline { 2 - 3 } Treatment & HL60 & HL60/VCR \\
\hline DOX & $67 \pm 11.0(1.00)^{\mathrm{b}}$ & $2,532.6 \pm 151(37.8)$ \\
+Butor 1 $(\mu \mathrm{M})$ & $65 \pm 9.0(0.97)$ & $1,326.6 \pm 232(19.8)^{\mathrm{c}}$ \\
+Butor 2 & $69 \pm 10.0(1.03)$ & $703.2 \pm 33.5(10.5)^{\mathrm{c}}$ \\
+Butor 4 & $60 \pm 8.0(0.92)$ & $506.5 \pm 35.6(7.56)^{\mathrm{c}}$ \\
+Verapamil 5 & $62 \pm 8.2(0.93)$ & $482.4 \pm 22.57(7.2)^{\mathrm{c}}$ \\
VCR & $2.7 \pm 0.8(1.00)^{\mathrm{b}}$ & $63.8 \pm 8.5(23.4)$ \\
+Butor 1 $(\mu \mathrm{M})$ & $2.58 \pm 0.75(0.95)$ & $28.43 \pm 3.80(10.53)^{\mathrm{c}}$ \\
+Butor 2 & $2.63 \pm 0.87(0.97)$ & $19.44 \pm 2.65(7.2)^{\mathrm{c}}$ \\
+Butor 4 & $2.85 \pm 0.61(1.06)$ & $12.96 \pm 1.53(4.8)^{\mathrm{c}}$ \\
+Verapamil 5 & $2.78 \pm 0.70(1.03)$ & $10.55 \pm 2.19(3.9)^{\mathrm{c}}$ \\
Cisplatin & $1,374 \pm 90.15(1.00)^{\mathrm{b}}$ & $1,701 \pm 94.16(1.23)$ \\
+Butor 1 $(\mu \mathrm{M})$ & $1,410 \pm 102.60(1.02)$ & $1,649 \pm 92.54(1.20)$ \\
+Butor 2 & $1,319 \pm 88.29(0.96)$ & $1,662 \pm 92.30(1.21)$ \\
+Butor 4 & $1,370 \pm 85.41(1.00)$ & $1,648 \pm 90.75(1.20)$ \\
+ Verapamil 5 & $1,358 \pm 190.13(0.99)$ & $1,607.6 \pm 90.57(1.17)$ \\
\hline
\end{tabular}

${ }^{a} \mathrm{IC}_{50}$ values are represented as mean $\pm \mathrm{SD}$ of three independent experiments performed in triplicate. ${ }^{\text {b}}$ The fold reversal of MDR (values given in parentheses) was calculated by dividing the $\mathrm{IC}_{50}$ values of substrate in drug-resistant cells in the absence or presence of inhibitor or HL60 cells with inhibitor, by the $\mathrm{IC}_{50}$ values of HL60 cells without inhibitor. ${ }^{c} \mathrm{p}<0.01$, significantly different from those obtained in the absence of inhibitor. ABCB1, ATP-binding cassette subfamily B member 1; MDR, multidrug resistance; SD, standard deviations Butor, butorphanol.

and $\mathrm{K} 562$ cells, the $\mathrm{IC}_{50}$ values of DOX and VCR were not significantly different in the presence or absence of buotorphanol. Thus, our results suggest that butorphanol sensitizes ABCB1-overexpressing cells to chemotherapeutic agents which are ABCB1 substrates.

Increased DOX accumulation by butorphanol in the HL60/VCR cells. To explore the potential mechanism of butorphanol in HL60/VCR and K562/ADR cells, we examined the intracellular accumulation levels of DOX in the HL60/VCR cells and counterpart HL60 cells, respectively. As shown in Fig. 2A and B, in the absence of butorphanol, the fluorescence of DOX was lower in the resistant cells than that in the sensitive cells. Yet, combined with 1,2 or $4 \mu \mathrm{M}$ butorphanol, accumulation levels of DOX in the HL60/VCR cells was obviously increased by 2.3-, 3.47- and 5.91-fold, respectively. The intracellular accumulation effect of DOX with $4 \mu \mathrm{M}$ of butorphanol was similar to that with $5 \mu \mathrm{M}$ of verapamil. However, neither butorphanol nor verapamil affected the intracellular levels of DOX in the HL60 cells. These results indicated that butorphanol increased the intracellular accumulation of DOX in the HL60/VCR cells in a concentration-dependent manner. 
A

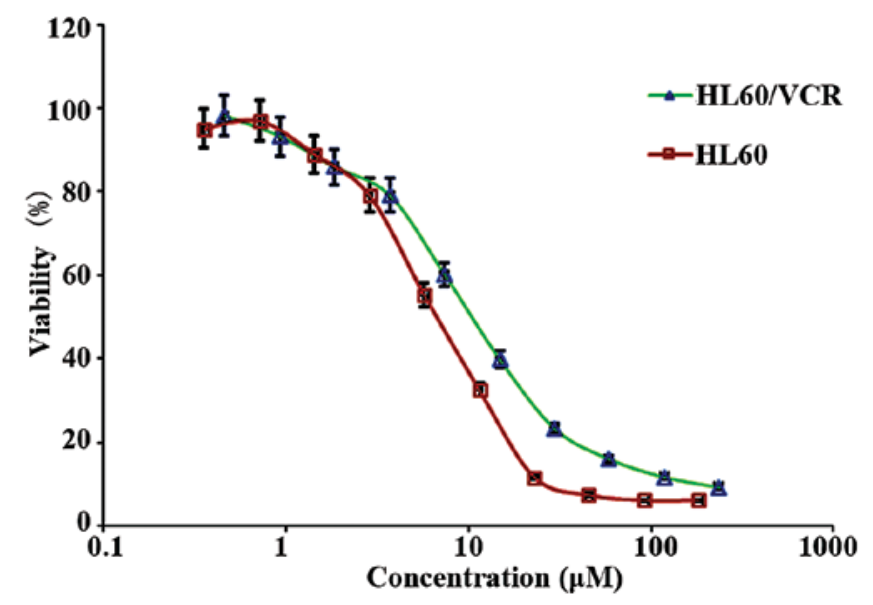

B

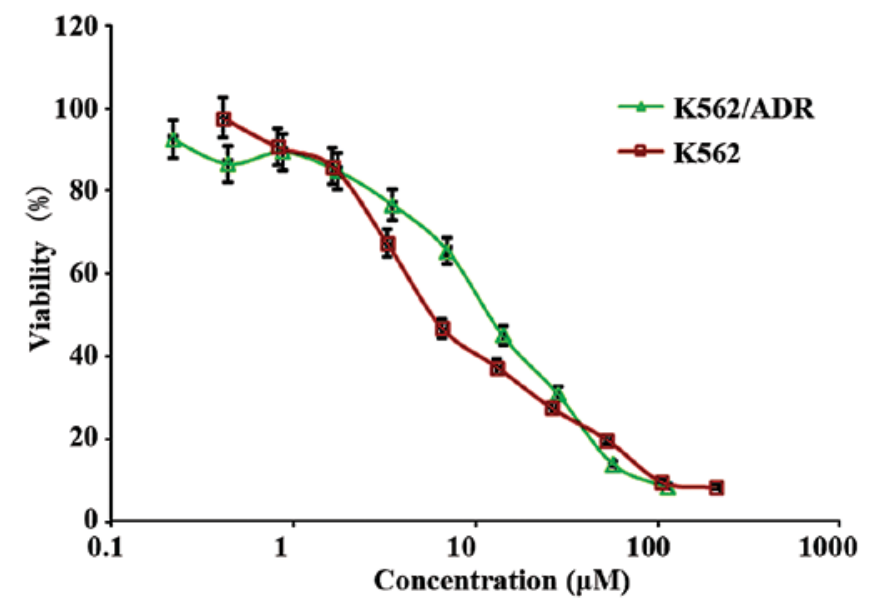

Figure 1. Cytotoxicity assays of butorphanol in parental and drug-resistant cells. The cell cytotoxicity was measured by MTT assay as described in Materials and methods in (A) HL60 and ABCB1-overexpressing HL60/VCR cells and (B) K562 and ABCB1-overexpressing K562/ADR cells. Data are expressed as the mean \pm standard deviation (SD) of triplicate independent determinations. Each experiment was repeated three times. ABCB1, ATP-binding cassette subfamily B member 1.

Effect of butorphanol on the expression levels and cellular localizations of $A B C B 1$. To evaluate whether butorphanol alters the protein expression of $\mathrm{ABCB} 1$, we examined the protein levels of $\mathrm{ABCB} 1$ following treatment with butorphanol at $4 \mu \mathrm{M}$ for $0,24,48$ and $72 \mathrm{~h}$ in the HL60/VCR and K562/ ADR cells. The protein levels of ABCB1 were not significantly altered after incubation with butorphanol at $4 \mu \mathrm{M}$ for up to $72 \mathrm{~h}$ (Fig. 3A-D). In addition, we also tested the localization of ABCB1 in the HL60/VCR cells in the presence of butorphanol when treated with the drug for up to $72 \mathrm{~h}$. There was no obvious modulation in regards to the surface localization of $\mathrm{ABCB} 1$ between treatment with or without butorphanol in the HL60/ VCR cells (Fig. 3E). These results indicated that the reversal of ABCB1-mediated MDR by butorphanol was neither through altered expression nor translocation of $\mathrm{ABCB} 1$.

Model of molecular docking for butorphanol binding with $A B C B 1$. To predict the binding conformation of the large cavity of the transmembrane region of $\mathrm{ABCB} 1$, we used a homology model of human ABCB1 to explore the potential binding
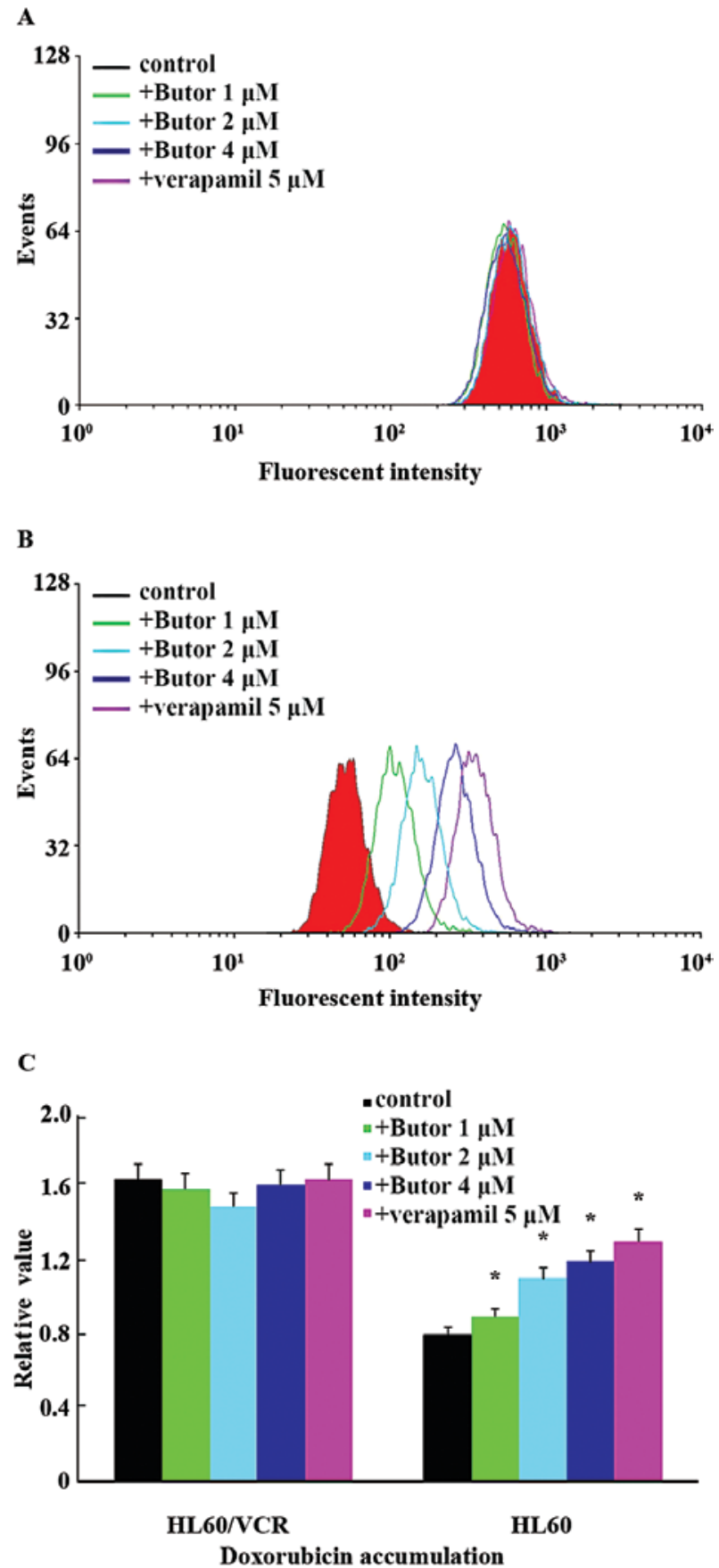

Figure 2. Effect of butorphanol on the accumulation of doxorubicin (DOX) (A and B) The accumulation of DOX in the HL60 and HL60/VCR cells was measured by flow cytometric analysis as described in Materials and methods. All experiments were repeated at least thrice. Data are represented as mean $\pm \mathrm{SD}$. ${ }^{*} \mathrm{p}<0.01$.

mode with Glide docking software. There are three binding sites in the homology model of ABCB1: ABCB1-QZ59-RRR (site-1), ABCB1-QZ59-SSS (site-2) and ABCB1-verapamil (site-3). The binding energy data (Glide scores for butorphanol at site-1 to site-3) suggest that site-1 is the most suitable one. The predicted docking conformation of butorphanol with the large hydrophobic drug binding cavity (site-1) of human $\mathrm{ABCB} 1$ is shown in Fig. 4A and B. The 3.14-morphinan group is stabilized into a hydrophobic pocket formed by residues IIe340, Met68, Phe75, Tyr307, Phe339, IIe343, Phe951, Phe981 and Va1987. Moreover, the 17-cyclobutylmethyl formed 
A

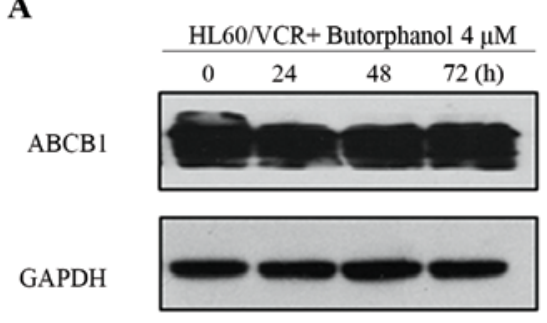

C

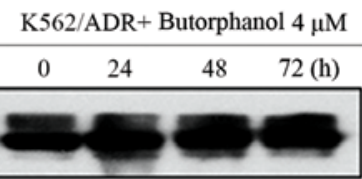

GAPDH

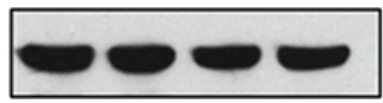

B

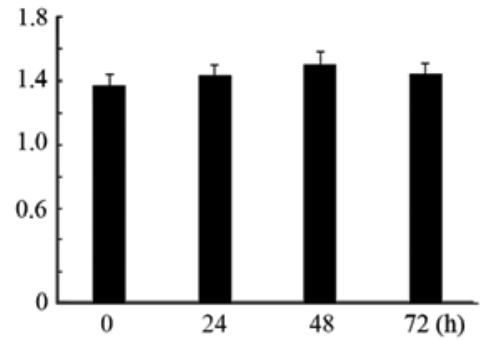

D

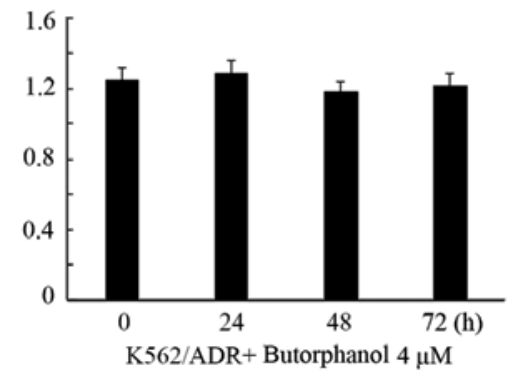

E HL60/VCR + Butorphanol $4 \mu \mathrm{M}(0 \mathrm{~h})$

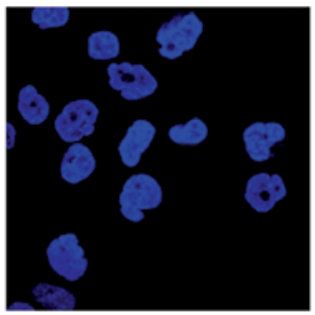

DAPI

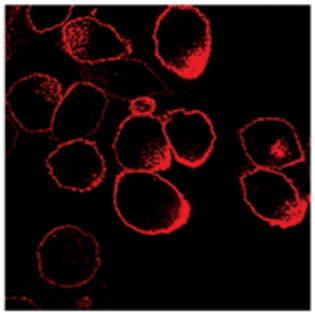

$\mathrm{ABCB} 1$

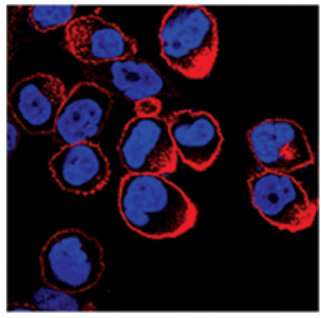

Merge

HL60/VCR + Butorphanol $4 \mu \mathrm{M}(72 \mathrm{~h})$

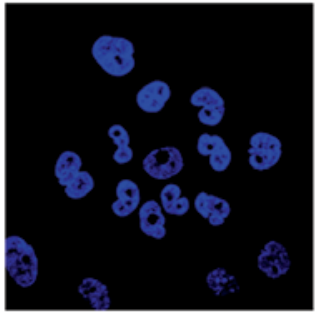

DAPI

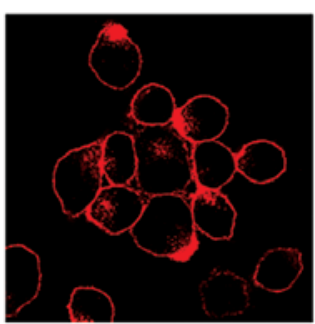

$\mathrm{ABCB} 1$

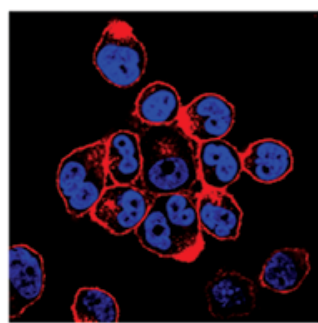

Merge

Figure 3. Butorphanol did not affect the protein expression level and localization of ABCB1 in the ABCB1-overexpression cells. (A and C) HL60/VCR cells were tested after treatment with butorphanol $4 \mu \mathrm{M}$ for $0,24,48$ and $72 \mathrm{~h}$ with western blotting, and (B and D) grayscale ratios of ABCB1 were analyzed with ImageJ. The grayscale ratios were proportional to ABCB1 protein levels. (E) Immunofluorescence staining was used to show the cellular localization of ABCB1 in the HL60/VCR cells after treatment with butorphanol for $72 \mathrm{~h}$. ABCB1 staining is shown in red. Fluorescent DAPI (blue) was used to counterstain the nuclei. ABCB1, ATP-binding cassette subfamily B member 1 .

hydrophobic bond interacted with the side chain of Phe339 and was stabilized through hydrophobic contact with the side chain of Met64, Phe335 and Ile330.

\section{Discussion}

In the present study, we first demonstrated that butorphanol, a kind of opioid analgesics, owns potentially antineoplastic function via reversal of ABCB1-mediated MDR in leukemia cell lines.

Over the past three decades, obvious developments have been achieved in the treatment options for leukemia and improvements in the clinical effects of anticancer drugs have been noted. Following standard first-line treatment, adults with leukemia have a $60-90 \%$ chance of attaining a completely hematologic remission (22). Unfortunately, the estimated 5-year survival of $40 \%$ is extremely low, since most patients subsequently suffer relapse after withdrawal of cytotoxic agents. Moreover, there is no effective therapy for patients with relapse (23). MDR has been known as the mainly contributing factor to treatment failure, and its involvement has become a primary cause of mortality and a formidable impediment to achieving long-term remission in leukemia (24). The universally recognized mechanism of 


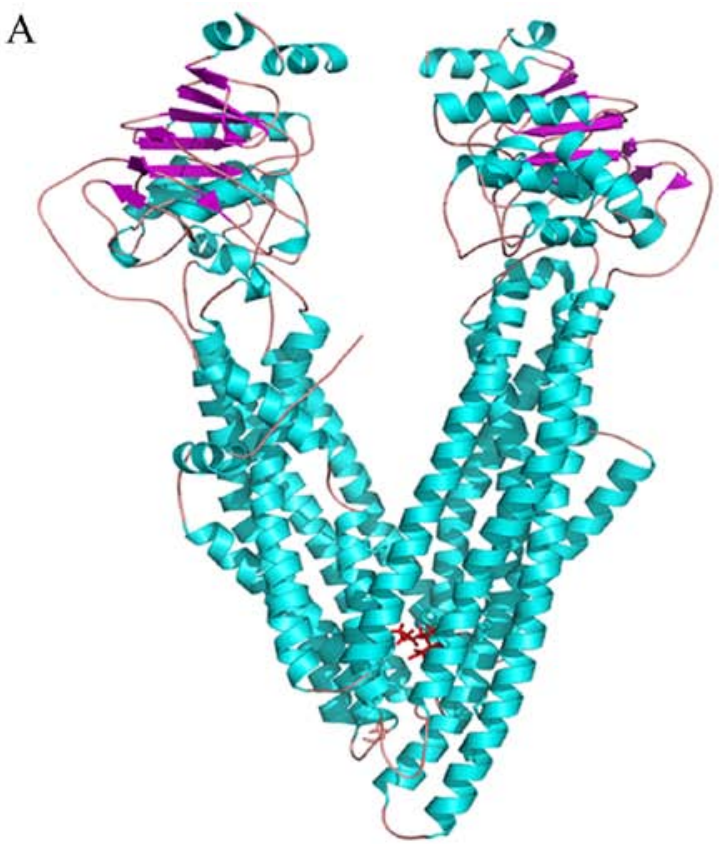

$\mathrm{B}$

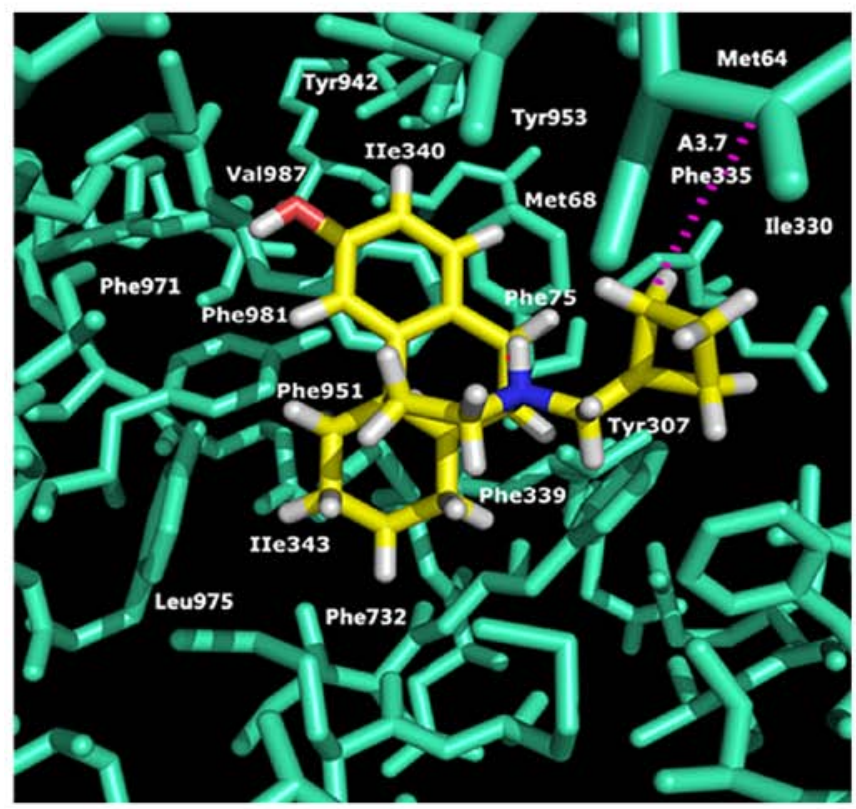

Figure 4. XP-Glide predicts the binding mode of butorphanol with homology modeled ABCB1. (A) Human ABCB1. (B) Binding mode of butorphanol within the drug binding site-1 of human ABCB1. Important amino acids are depicted as sticks with the atoms colored carbon-green, hydrogen-white, nitrogen-blue, oxygen-red and sulfur-yellow; carbon atoms are represented in green. The dotted red line indicates electrostatic interactions. The ABCB1 model is represented as MacroModel surface based on residue charge (hydrophobic-green). ABCB1, ATP-binding cassette subfamily B member 1.

MDR may be the overexpression of ABC transporters which can weaken the cytotoxic effect of chemotherapeutic agents via transferring drugs to the cell exterior (25). A clinical metaanalysis of 1,826 patients found that an increased ABCB1 level in leukemia patients confers a worse response to conventional anticancer drugs (26). Recent studies have also identified that leukemia cells with high levels of ABCB1 produce stubborn resistance to imatinib which represents a standard regimen for the first-line treatment of leukemia (27). Thus, ABCB1 may be a potential target for successful chemotherapy of leukemia and a combination of ABCB1 inhibitors together with the chemotherapeutic regimen may be an effective strategy.

Over the last decade, a large number of ABCB1 inhibitors have been developed and are categorized into three generations based on sequential refinements in pharmacodynamic properties (28). Yet, most are limited in regards to their application in the clinic due to serious side-effects and unexpected interaction affecting pharmacokinetics (29). Therefore, the development of safe and effective ABCB1 inhibitors to overcome MDR is critical. Identification of previously approved drugs for use as chemotherapeutic sensitizers is a more efficient method which has been discussed broadly.

In the present study, we demonstrated that butorphanol reversed ABCB1-mediated MDR in leukemia cell lines at clinical doses by promoting the penetration of chemotherapeutic agents into tumor cells, increasing their bioavailability and achieving a better treatment outcome in leukemia cells. As a type of synthetic opioid, butorphanol is applied to alleviate moderate to serious pain, such as intraoperative, postoperative and malignant trauma pain (30). Butorphanol is used to produce an intrinsic function in the treatment of leukemia $(31,32)$. Based on these finding, butorphanol has the ability to overcome MDR in parallel with the suppression of pain in leukemia patients.

To evaluate the sufficient concentration and cytotoxicity of butorphanol, we identified the maximum reversing concentration of butorphanol for combination treatment with antineoplastic drugs by MTT assay (Fig. 1A and B) (33). The results of the MTT assay showed that butorphanol at $4 \mu \mathrm{M}$ did not restrain the growth of the cells used in the present study. The results of colony formation assay were consistent with the MTT assay (data not shown). Thus, butorphanol itself had no cytotoxic effect on the leukemia cells. As shown in Tables I and II, butorphanol had no additive or synergistic antitumor effect in sensitive cells, but in the resistant cells, butorphanol markedly increased the sensitivity to DOX and VCR of the HL60/VCR and K562/ADR cells, respectively. Compared with the saline group, the resistance fold was effectively reduced to 7.5 -fold by $4 \mu \mathrm{M}$ butorphanol; the resistance rate was decreased by $76.4 \%$ compared with the group without butorphanol. The reversal effect of butorphanol was achieved in a dose-dependent manner.

The obvious results induced us to investigate the reversal mechanism of butorphanol in the leukemia cells. We tested the drug accumulation of DOX in the HL60/VCR cells. The result of flow cytometric analyses demonstrated that butorphanol significantly increased the intracellular accumulation of Dox (Fig. 2A and B). Similar effects were not found in the parental HL60 cells (15). These observations were consistent with previous studies (34). Some studies have identified that there are two main ways leading to the reversal of MDR, reducing $\mathrm{ABCB} 1$ expression or inhibiting the efflux function of ABCB1 (35). Therefore, we examined the ABCB1 expression by treating the $\mathrm{ABCB}$-overexpressing cells with butorphanol at $4 \mu \mathrm{M}$ for 24,48 and $72 \mathrm{~h}$, respectively. The results showed that butorphanol did not alter the ABCB1 protein expression in the HL60/VCR and K562/ADR cells (Fig. 3A-D). In addition, there was no alteration in $\mathrm{ABCB} 1$ protein expression in 
the plasma membranes of the HL60/VCR cells after treatment with butorphanol (Fig. 3E). A number of recent studies have corroborated that numerous $\mathrm{ABC}$ modulators directly inhibit the pump function of $\mathrm{ABC}$ transporters without changing their protein expression levels, such as motesanib (36), AST1306 (37) and tivozanib (38). Notably, Hamabe et al reported that morphine, fentanyl and methadone can increase ABCB1 ATPase activity, and their concentration-responses are bell-shaped (39). Gonzalez et al also demonstrated that the ATPase activity was obviously higher in cells with low concentration of morphine than cells with morphine tolerance (40). As an analogue of morphine, the structure and function of butorphanol are similar to morphine; thus, it may also have similar effects. Butorphanol at low concentrations competitively binds to the sites of ABCB1, leaving few sites for transporter substrates. When its concentration is beyond a threshold, butorphanol directly inhibits ATPase activity (7). Thus, we conclude that butorphanol potentiated the sensitivity of anticancer agents in HL60/VCR and K562/ADR cells through directly inhibiting the efflux function of ABCB1.

To further understand the mechanisms underlying the ABCB1 inhibitor butorphanol, we tested the docking simulation of butorphanol and ABCB1 (21). Through docking score, we identified the most appropriate binding site. Electrostatic interaction of $\mathrm{NH}$ may form correct and stable conformations in the hydrophobic pocket of ABCB1. Cyclobutylmethyl and morphinan provide the predominant pharmacophoric features for docking at the ABCB transporter. Considering the above findings, butorphanol may be a better candidate as an ABCB1 inhibitor in leukemia cells via providing a more effective and suitable method to prevent and correct ABCB1-mediated MDR, simultaneously suppressing cancer-related pain and enhancing the quality of life of these patients.

In conclusion, the discovery of butorphanol offers a potential method of the pharmacologic downregulation of ABCB1-mediated MDR in HL60/VCR and K562/ADR cells in vitro. These results suggest that butorphanol may be a new candidate as an ABCB1 inhibitor and may be combined with conventional anticancer drugs to overcome MDR. Butorphanol may also simultaneously improve the pain threshold of patients.

However, before butorphanol can be administered to leukemia patients further investigations are needed. Yet, the present study provides an important clue to explore new functions of drugs that have been previously used in the clinic.

\section{References}

1. Cooper SL and Brown PA: Treatment of pediatric acute lymphoblastic leukemia. Pediatr Clin North Am 62: 61-73, 2015.

2. Lussana F and Rambaldi A: Role of allogeneic hematopoietic stem cell transplantation in adult patients with acute lymphoblastic leukemia. Mediterr J Hematol Infect Dis 6: e2014065, 2014.

3. te Boekhorst PA, de Leeuw K, Schoester M, Wittebol S, Nooter K, Hagemeijer A, Löwenberg B and Sonneveld P: Predominance of functional multidrug resistance (MDR-1) phenotype in CD34+ acute myeloid leukemia cells. Blood 82: 3157-3162, 1993.

4. Zhang BB, Xuan C, Deng KF, Wu N and Lun LM: Association between the $M D R 1$ gene variant $\mathrm{C} 3435 \mathrm{~T}$ and risk of leukaemia: A meta-analysis. Eur J Cancer Care 22: 617-625, 2013.

5. Lage $\mathrm{H}$ : An overview of cancer multidrug resistance: A still unsolved problem. Cell Mol Life Sci 65: 3145-3167, 2008.

6. Saneja A, Khare V, Alam N, Dubey RD and Gupta PN: Advances in P-glycoprotein-based approaches for delivering anticancer drugs: Pharmacokinetic perspective and clinical relevance. Expert Opin Drug Deliv 11: 121-138, 2014.
7. Gillet JP, Efferth T and Remacle J: Chemotherapy-induced resistance by ATP-binding cassette transporter genes. Biochim Biophys Acta 1775: 237-262, 2007.

8. Shi Z, Tiwari AK, Shukla S, Robey RW, Kim IW, Parmar S, Bates SE, Si QS, Goldblatt CS, Abraham I, et al: Inhibiting the function of $\mathrm{ABCB} 1$ and $\mathrm{ABCG} 2$ by the EGFR tyrosine kinase inhibitor AG1478. Biochem Pharmacol 77: 781-793, 2009

9. Du BX, Song ZM, Wang K, Zhang H, Xu FY, Zou Z and Shi XY: Butorphanol prevents morphine-induced pruritus without increasing pain and other side effects: A systematic review of randomized controlled trials. Can J Anaesth 60: 907-917, 2013.

10. Parikh GP, Veena SR, Vora K, Parikh B and Joshi A: Comparison of epidural butorphanol versus epidural morphine in postoperative pain relief. Middle East J Anaesthesiol 22: 371-376, 2014.

11. Shah B, Raichandani Y and Misra A: Development and evaluation of oral osmotic pump of butorphanol tartrate. Pharm Dev Technol 19: 868-880, 2014.

12. Guo HQ, Zhang GN, Wang YJ, Zhang YK, Sodani K, Talele TT, Ashby CR Jr and Chen ZS: $\beta$-elemene, a compound derived from Rhizoma zedoariae, reverses multidrug resistance mediated by the ABCB1 transporter. Oncol Rep 31: 858-866, 2014.

13. Xie X, Yin J, Jia Q, Wang J, Zou C, Brewer KJ, Colombo C, Wang Y, Huang G and Shen J: Quercetin induces apoptosis in the methotrexate-resistant osteosarcoma cell line U2-OS/MTX300 via mitochondrial dysfunction and dephosphorylation of Akt. Oncol Rep 26: 687-693, 2011

14. Bonelli MA, Fumarola C, Alfieri RR, La Monica S, Cavazzoni A, Galetti M, Gatti R, Belletti S, Harris AL, Fox SB, et al: Synergistic activity of letrozole and sorafenib on breast cancer cells. Breast Cancer Res Treat 124: 79-88, 2010.

15. Zhao T, Song Y, Liu B, Qiu Q, Jiao L, Li Y, Huang W and Qian H: Reversal of P-glycoprotein-medicated multidrug resistance by LBM-A5 in vitro and a study of its pharmacokinetics in vivo. Can J Physiol Pharmacol 93: 33-38, 2015.

16. Zhou WJ, Zhang X, Cheng C, Wang F, Wang XK, Liang YJ, To KK, Zhou W, Huang HB and Fu LW: Crizotinib (PF-02341066) reverses multidrug resistance in cancer cells by inhibiting the function of P-glycoprotein. Br J Pharmacol 166: 1669-1683, 2012.

17. Fu LW, Zhang YM, Liang YJ, Yang XP and Pan QC: The multidrug resistance of tumour cells was reversed by tetrandrine in vitro and in xenografts derived from human breast adenocarcinoma MCF-7/adr cells. Eur J Cancer 38: 418-426, 2002.

18. Kim SK, Jung WH and Koo JS: Differential expression of enzymes associated with serine/glycine metabolism in different breast cancer subtypes. PLoS One 9: e101004, 2014.

19. Samuel S, Beljanski V, Van Grevenynghe J, Richards S, Ben Yebdri F, He Z, Nichols C, Belgnaoui SM, Steel C, Goulet ML, et al: BCL-2 inhibitors sensitize therapy-resistant chronic lymphocytic leukemia cells to VSV oncolysis. Mol Ther 21: 1413-1423, 2013.

20. Aggarwal H, Aggarwal A, Hunter WJ III, Yohannes P, Khan AU and Agrawal DK: Expression of leukemia/lymphoma related factor (LRF/Pokemon) in human benign prostate hyperplasia and prostate cancer. Exp Mol Pathol 90: 226-230, 2011.

21. Li X, Duan S, Chu C, Xu J, Zeng G, Lam AK, Zhou J, Yin Y, Fang D, Reynolds MJ, et al: Melaleuca alternifolia concentrate inhibits in vitro entry of influenza virus into host cells. Molecules 18: 9550-9566, 2013.

22. Berman E, Heller G, Santorsa J, McKenzie S, Gee T, Kempin S, Gulati S, Andreeff M, Kolitz J, Gabrilove J, et al: Results of a randomized trial comparing idarubicin and cytosine arabinoside with daunorubicin and cytosine arabinoside in adult patients with newly diagnosed acute myelogenous leukemia. Blood 77: 1666-1674, 1991.

23. Pulte D, Gondos A and Brenner H: Expected long-term survival of patients diagnosed with acute myeloblastic leukemia during 2006-2010. Ann Oncol 21: 335-341, 2010.

24. Vargas JR, StanzlEG, Teng NN and Wender PA: Cell-penetrating, guanidinium-rich molecular transporters for overcoming effluxmediated multidrug resistance. Mol Pharm 11: 2553-2565, 2014.

25. Nori A and Kopecek J: Intracellular targeting of polymer-bound drugs for cancer chemotherapy. Adv Drug Deliv Rev 57: 609-636, 2005.

26. Zheng Q, Wu H, Yu Q, Kim DH, Lipton JH, Angelini S, Soverini S, Vivona D, Takahashi N and Cao J: ABCB1 polymorphisms predict imatinib response in chronic myeloid leukemia patients: A systematic review and meta-analysis. Pharmacogenomics J 15: 127-134, 2015.

27. Kosztyu P, Bukvova R, Dolezel P and Mlejnek P: Resistance to daunorubicin, imatinib, or nilotinib depends on expression levels of ABCB1 and ABCG2 in human leukemia cells. Chem Biol Interact 219: 203-210, 2014. 
28. Shaffer BC, Gillet JP, Patel C, Baer MR, Bates SE and Gottesman MM: Drug resistance: Still a daunting challenge to the successful treatment of AML. Drug Resist Updat 15: 62-69, 2012.

29. Krishna R, St-Louis M and Mayer LD: Increased intracellular drug accumulation and complete chemosensitization achieved in multidrug-resistant solid tumors by co-administering valspodar (PSC 833) with sterically stabilized liposomal doxorubicin. Int J Cancer 85: 131-141, 2000 .

30. Golianu B, Krane EJ, Galloway KS and Yaster M: Pediatric acute pain management. Pediatr Clin North Am 47: 559-587, 2000.

31. Osipova NA, Petrova VV, Novikov GA, Ziai GR and Mel'nikova ZL: Synthetic analgesic moradol at various stages of surgical treatment of patients with cancer. Anesteziol Reanimatol 2: 56-58, 1990 (In Russian).

32. Nakadate H, Endoh M, Satake A, Sida S, Hatayama Y, Hatae Y, Takeda T, Wada I, Itho T and Kidoguchi T: Continuous infusion of butorphanol for pain in children with cancer. Gan To Kagaku Ryoho 16: 3495-3498, 1989 (In Japanese).

33. Shi Z, Tiwari AK, Shukla S, Robey RW, Singh S, Kim IW Bates SE, Peng X, Abraham I, Ambudkar SV, et al: Sildenafil reverses ABCB1- and ABCG2-mediated chemotherapeutic drug resistance. Cancer Res 71: 3029-3041, 2011.

34. Sodani K, Patel A, Anreddy N, Singh S, Yang DH, Kathawala RJ, Kumar P, Talele TT and Chen ZS: Telatinib reverses chemotherapeutic multidrug resistance mediated by ABCG2 efflux transporter in vitro and in vivo. Biochem Pharmacol 89: 52-61, 2014.
35. Wang XK, To KK, Huang LY, Xu JH, Yang K, Wang F, Huang ZC, Ye $S$ and Fu LW: Afatinib circumvents multidrug resistance via dually inhibiting ATP binding cassette subfamily G member 2 in vitro and in vivo. Oncotarget 5: 11971-11985, 2014.

36. Wang YJ, Kathawala RJ, Zhang YK, Patel A, Kumar P, Shukla S, Fung KL, Ambudkar SV, Talele TT and Chen ZS: Motesanib (AMG706), a potent multikinase inhibitor, antagonizes multidrug resistance by inhibiting the efflux activity of the ABCB1. Biochem Pharmacol 90: 367-378, 2014.

37. Zhang H, Wang YJ, Zhang YK, Wang DS, Kathawala RJ, Patel A, Talele TT, Chen ZS and Fu LW: AST1306, a potent EGFR inhibitor, antagonizes ATP-binding cassette subfamily G member 2-mediated multidrug resistance. Cancer Lett 350: 61-68, 2014

38. Yang D, Kathawala RJ, Chufan EE, Patel A, Ambudkar SV, Chen ZS and Chen X: Tivozanib reverses multidrug resistance mediated by ABCB1 (P-glycoprotein) and ABCG2 (BCRP). Future Oncol 10: 1827-1841, 2014.

39. Hamabe W, Maeda T, Fukazawa Y, Kumamoto K, Shang LQ, Yamamoto A, Yamamoto C, Tokuyama S and Kishioka S: P-glycoprotein ATPase activating effect of opioid analgesics and their P-glycoprotein-dependent antinociception in mice. Pharmacol Biochem Behav 85: 629-636, 2006.

40. GonzalezLG, Masocha W,Sánchez-Fernández C, Agil A, Ocaña M, Del Pozo E and Baeyens JM: Changes in morphine-induced activation of cerebral $\mathrm{Na}^{+}, \mathrm{K}^{+}$-ATPase during morphine tolerance: Biochemical and behavioral consequences. Biochem Pharmacol 83: 1572-1581, 2012. 